\title{
La Minustah y la reconstrucción del Estado haitiano*
}

\author{
Juan Gabriel Valdés ${ }^{\star}$
}

\section{INTRODUCCIÓN}

Una Misión de Paz de las Naciones Unidas genera, como toda intervención externa, un choque de percepciones e ideologías. No habrá nunca una coincidencia completa entre las visiones de quienes han recibido el mandato de intervenir y aquella que tienen de sí mismos los ciudadanos de la nación intervenida. Por más que los primeros practiquen el respeto y la dedicación, la realidad intervenida les resultará siempre enigmática y el desarrollo de su tarea será arduo; por más disposición que muestren los segundos hacia la intervención, siempre habrá en ellos una herida en sus sentimientos de dignidad nacional, junto a la idea de que hay un error básico en la percepción que el otro tiene del país. Pronto surgirá en algunos locales la tentación de descalificar la comprensión de los foráneos.
De utilizar la caricatura o la diatriba como un arma arrojadiza contra sus interventores ${ }^{1}$.

1 Un buen ejemplo, son las desaforadas acusaciones que, en su prefacio al libro de Sauveur Pierre Etienne "L'enigme haitienne», de reciente publicación, lanza el profesor Laennec Hurbon, Director de Investigaciones del CNRS de Paris. Dice Hurbon que reconocer la crisis del Estado haitiano y su mala conformación histórica, «está lejos de consagrar la tesis intervencionista del chileno Juan Gabriel Valdés, jefe político de la Misión de las Naciones Unidas para la Estabilización de Haiti (Minustah) que, bajo el pretexto de la ingobernabilidad de Haití, propone como solución una nueva forma de colonización». "Una colonización que, a decir verdad», dice el autor "se desarrolla enmascarando una complacencia insidiosa hacia las bandas armadas de las poblaciones marginales, cuando no se alimenta de principios que han hecho la fortuna de las dictaduras de Duvalier y de Aristide, a saber la reactivación del esquema simplis-

* Ex representante especial del Secretario General de las Naciones Unidas en Haití, jefe de la MINUSTAH (jvaldes@aol.com).

* Una versión más completa de este artículo será publicada por la Universidad Torcuato di Tella, de Buenos Aires, bajo la dirección de Mónica Hirst. 
De la misma manera, por muy desprejuiciados que sean los que intervienen, siempre se filtrarán en su perspectiva las preocupaciones e ideologías que traen consigo. Necesariamente arrastrarán el peso y el valor de sus experiencias nacionales, de sus propios conflictos internos, de sus construcciones políticas. Y quienes les reciben levantarán como bandera, en parte como consecuencia, la idea de que las soluciones que les son propuestas son ajenas, ya sea porque sienten que los que las hacen no tienen derecho a hacerlas, o bien porque las creen extrañas a su singular y excepcional construcción histórica. En consecuencia, por más neutralidad que aleguen quienes intervienen, y abiertos hacia su acción sean los intervenidos, el intercambio de acusaciones de parcialidad por una parte y de obcecación por la otra estará siempre a flor de labios.

Estas consideraciones -importantes como son- no deberían resultar en impedimentos para actuar. Porque si los que intervienen se aplican de verdad a promover la reconstitución de una legitimidad interna, los alegatos contrarios se diluirán y habrá una mayoría dispuesta a dar los pasos necesarios para recuperar su soberanía, usando

ta de la oposición Negros/Mulatos, como si todos los males del país fueran producidos y mantenidos por un grupo de «mulatos» y de «burgueses», y sobre todo, como si ella, la llamada comunidad internacional, pudiera súbitamente ser poseída por un amor sin límites por los (pobres) Negros de Haití». en el camino la asistencia de los que acudieron en nombre de la comunidad internacional.

Estas reflexiones pueden ser útiles en el análisis de un caso como el de Haití y de la más reciente de las operaciones internacionales en ese país. Primero, por las extraordinarias características de esa nación, donde la compleja relación de una identidad heroica y libertaria con una lista larga de intervenciones extranjeras genera en la población actitudes ambivalentes hacia el extranjero. Enseguida, porque esta última intervención internacional en Haití fue autorizada por el Consejo de Seguridad en el marco del Capítulo VII de la Carta de las Naciones Unidas, que facultaba a la Misión a usar la fuerza dentro de un Estado soberano, miembro de las Naciones Unidas, donde no había propiamente una guerra, sino un enfrentamiento entre facciones a la larga minoritarias y el peligroso pulular de bandas criminales financiadas por los intereses de la droga. Pero a la vez, porque la Misión -a pesar de sus atribuciones de guerra interna- estaba condicionada por el Consejo de Seguridad a actuar «en apoyo» de un gobierno provisional, es decir, a operar en muchas áreas vitales para el éxito de la operación, de manera subsidiaria a un gobierno que actuaba prácticamente en ausencia de un Estado, carecía de legitimidad democrática y tenía graves problemas de unidad interna. Todo eso hizo indudablemente más compleja la relación de interventores e intervenidos. 
Pero si estas características adquirían en el caso haitiano una relevancia particular, era principalmente porque el conflicto interno que se pretendía reducir revestía en la práctica un carácter esencialmente político y solo muy secundariamente un carácter militar. De esta manera, el cruce de percepciones sobre el sentido político de la intervención se transformó desde muy pronto en la cuestión central que debía ser resuelta, y fue el terreno principal de interacción y conflicto entre los actores internos y externos. Así, la esencia del problema de la Misión de Paz -en su inicio- radicó en la orientación política que se pretendía dar al proceso de transición. Y tan importante fue conocer la fuerza militar de que disponían los violentos, como fue comprender las intenciones de los actores políticos respecto de aquellos, las señales que emitían respecto de la estabilización de la sociedad y la interpretación de «las máscaras» adoptadas en distintos momentos del proceso de interacción de las élites locales con la fuerza interventora.

Esta cuestión es central. Plantea en términos generales la complejidad que adquiere el rol político de los mandos de una Misión de Paz, y la relación entre las tareas de naturaleza política $\mathrm{y}$ aquellas de naturaleza militar y policial que caracterizan en principio este tipo de operaciones internacionales. Se vincula directamente con el dilema de la relación entre la urgencia de detener la violencia en el menor tiempo posible y de intentar comprender y luego desac- tivar aquellas cuestiones de fondo que se hallan tras el caos que se pretende controlar: esencialmente el desempleo, la miseria y el abandono de millones de personas. Y la manera en que se planteen estos temas será determinante para el tipo de relación que se establece con las élites locales, con los intereses y las percepciones ideológicas con las que estas construyen su realidad.

Sucede que necesariamente algunos miembros de la élite considerarán más ofensivo hacia sus sentimientos nacionales el que la Misión «intervenga en política», que la propia presencia de tropas en el territorio. Tras intentar por todos los medios de atraerla hacia sus intereses de grupo, concluirán que la Misión se ha equivocado y su concepción es errada, incluso peligrosa. Algunos imaginarán toda suerte de hipótesis conspirativas. Otros se restarán de cualquier esfuerzo que promueva la Misión. Pero algunos comprenderán -como dijera al jefe de la Misión un destacado dirigente político- que si bien las Naciones Unidas no pueden cambiar el país porque ello solo pueden hacerlos los haitianos, al menos pueden impedir que suceda lo peor. Y que para lograr este objetivo, la acción de la fuerza militar contribuye, pero no reemplaza la persuasión y la paciente búsqueda común de caminos políticos y de desarrollo. Es decir, la fuerza no reemplaza a la política ${ }^{2}$.

2 Agradeceré siempre a Marc Bazin la primera conversación, la más importante de las muchas que tuvimos en la casa de Bourdon. 
Pero, ¿qué caminos proponen o alientan quienes vienen como «enviados» a un país cuyos contenidos culturales conocen solo de manera textual? Naturalmente -se dirá- aquellos establecidos en las resoluciones del Consejo de Seguridad. Sin embargo, los llamados del Consejo de Seguridad a «estabilizar el país»; ayudar el desarrollo de «la transición»; apoyar la organización de elecciones; contribuir a la reconciliación entre los haitianos, promover «el diálogo nacional», constituyen todas ellas tareas que requieren de juicio político. Para los latinoamericanos, involucrados desde un inicio y de manera determinante en la composición de la Misión, estos conceptos no podían sino resultar conocidos, visto el desarrollo tan reciente de procesos políticos de transición política en el área. Por ello, las estrategias de implementación de estos conceptos, los límites de lo que era juzgado posible o imposible, el clima político requerido para avanzar en la tarea y los tiempos disponibles para ello,-decisiones que fueron necesariamente confiadas a quienes conducían la misión-, se hallaban mediadas necesariamente por sus experiencias.

Esto plantea preguntas de mayor envergadura: ¿hasta qué punto el predominio de militares latinoamericanos entre los efectivos de la Minustah, el nombramiento de latinoamericanos en la conducción de la Misión, y el compromiso de los gobiernos latinoamericanos con ella, estableció diferencias con las intervenciones previas de países u organismos internacionales en Haití?
Más aún, ¿̇hasta qué punto la forma en que fue interpretado y "comprendido" el proceso político haitiano correspondió a la transposición de experiencias latinoamericanas? ¿No hay acaso un conjunto de reacciones típicas en la región ante problemas como «desarrollo», «transición», «derechos humanos», política y elecciones?

Estas notas no pretenden responder exhaustivamente estas preguntas, algo que requeriría sin dudas de una extensión mayor. Constituyen sin embargo una primera aproximación al tema de las características políticas de la Misión en Haití hasta la elección de René Preval como Presidente constitucional de la República en febrero de 2006.

\section{La política de la Minustah}

La construcción de la política de la Misión incluyó en principio tres nociones centrales estrechamente vinculadas entre sí. La primera provenía de un principio que no solo se hallaba inscrito en el mandato, sino también, y de manera muy particular, en la experiencia histórica de quienes debían aplicarlo: el del respeto por los derechos humanos de todos los haitianos, pero especialmente de quienes sufrían la violencia interna. Se puede comprender que mal podía ser este un tema secundario para soldados y civiles latinoamericanos.

Él planteaba sin dudas un dilema a quienes conducían la Misión. Implicaba por una parte no llevar a cabo operaciones de carácter militar en las pobla- 
ciones marginales que subordinaran los derechos humanos de sus habitantes al objetivo de obtener victorias ante los violentos. Era evidente para todos, y muy especialmente para los criminales, que el ingreso ofensivo de tropas de infantería a una población de varios centenares de miles de habitantes, hacinados en casuchas de material ligero, arriesgaba producir enormes costos humanos en la población civil. Los violentos sabían arroparse en niños y mujeres, asegurándose así que su captura o su muerte conduciría necesariamente a la destrucción de la legitimidad de la Misión en el país y en el exterior. En Miami, Nueva York y otras partes, un buen número de asociados de Aristide, dotados de contactos sorprendentemente efectivos en los medios liberales de comunicación, estaban siempre prontos a denunciar como "genocidio» cualquier intento de represión de las organizaciones armadas que ellos habían contribuido a formar.

Este dilema preocupaba muy especialmente los militares que conformaban la Misión. Tal como dijera un general argentino a una autoridad haitiana: «los militares latinoamericanos hemos pagado un costo terrible por las violaciones de los derechos humanos en nuestros países. No nos pidan aquí que vengamos a violar los derechos humanos en el suyo. No hemos venido a eso» ${ }^{3}$. Sin embargo, ¿cómo podían las autoridades de la Misión usar la fuerza

El General Eduardo Lugani a Bernard Gousse, Ministro de Justicia de Haití. a la que legítimamente estaban autorizados? ¿Cómo definían una estrategia que les permitiera mantener el principio básico de respeto a los derechos humanos y cumplir su misión de reducir a los violentos?

Tal como se encargaron de recordar con comprensible impaciencia los grupos de poder y las élites, la Misión debía proteger también los derechos de las víctimas que habitaban en otros lugares de la capital, y que sufrían secuestros, asaltos y otros desmanes en las incursiones de los bandas armadas por Puerto Príncipe. El incremento durante algunos períodos de los secuestros y los atentados en torno a Cité Soleil pronto transformaron estas demandas en acusaciones contra la Misión y en verdaderas campañas de radio y publicidad callejera destinadas a presionar a las autoridades civiles y militares de la Minustah. En ellas se exigía el uso del poder militar contra las bandas armadas de Cité Soleil y las demás poblaciones de la capital bajo la hipótesis de que cualquier proyecto político de reconstrucción institucional pasaba primero por «una limpieza» de los grupos de delincuentes que amenazaban a la población.

Sin duda que la suma de operaciones militares desarrolladas contra los violentos durante el primer año de la Minustah. Fue mucho más efectiva de lo que los críticos estaban dispuestos a reconocer. El famoso grupo de ex militares haitianos, que ocupó la atención del contingente internacional durante parte de ese año, fue desarticulado sin 
mayores costos humanos. Las bandas armadas perdieron a un número importante de sus líderes y vieron sus organizaciones cercadas y permanentemente amenazadas, en lo que fue una primera fase de su progresivo aislamiento del resto de la población. Los 90 policías haitianos y los 12 cascos azules muertos en el período previo a la elección; los 91 militares de Minustah heridos en enfrentamientos con los bandidos, muestran el alto costo pagado por Haití y por la Misión en la lucha por estabilizar el país. Sin embargo, la búsqueda de estrategias militares para aislar, cortar los suministros, dividir y reprimir a las bandas dio siempre resultados ambivalentes. La violencia de la sociedad haitiana se expandía más allá de las bandas armadas, era anómala y dispersa y difícil de asir en una estrategia militar. Pero no solo eso: la razón de fondo era que el problema no estaba ni principal ni solamente en la adecuación o inadecuación de las estrategias militares. El nudo del problema de la violencia radicaba esencialmente en el contexto político en que estas estrategias podian o no podían llevarse a cabo. Dicho de otra manera, las condiciones políticas del país eran determinantes para que las operaciones militares dieran los resultados deseados por el pueblo haitiano y la comunidad internacional.

De aquí deriva una segunda cuestión central en la política de la Misión. El objetivo de eliminar las bandas armadas no era posible si no se lograba debilitar sustantivamente la relación que ellas tenían con la población de los barrios más pobres. Y a su vez, esto solo podría lograrse en un escenario de mayor legitimidad estatal, en la que, en primer lugar, las acciones de la Minustah contra las bandas y en favor de la población, fueran ordenadas por (y coordinadas con) un gobierno haitiano legítimamente constituido. Se requería además que ese gobierno haitiano restituyera los vínculos mínimos de la autoridad estatal con la población más pobre de la ciudad. La partida de Aristide, que durante años había desarrollado una política clientelista con esos sectores, había cortado bruscamente todo vínculo del Estado hacia ellos, abriendo espacios que habían sido rápidamente ocupados por los violentos.

La urgencia de reconstruir y otorgar legitimidad a la policía haitiana, que la Misión halló en condiciones de práctica inexistencia, así como de recomponer los lazos del Estado con esas poblaciones, era una prioridad política de primer orden, que no podía tener soluciones automáticas. Requería de tiempo, y sobre todo, de la constitución por la sociedad haitiana de una base propia y soberana de poder que luchara por el aislamiento y la exclusión de los violentos. De aquí se deduce que para la Minustah la organización de elecciones legítimas, participativas y con resultados aceptados por todos, fuera siempre una prioridad superior. La propia estabilización y pacificación de la sociedad haitiana dependía de ellas.

Por último, era claro para quienes llegaban con la Misión que los niveles de 
pobreza y abandono en la que subsistían millones de haitianos en la capital y las principales ciudades del país constituía un factor «reproductor» de la violencia que por sí solo -incluso si hubiese sido posible dejar de lado cualquier consideración humanitaria hacia la poblaciónhacía inoperante cualquier estrategia de «limpieza» represiva como la que predicaban algunos sectores en el país. En un contexto de extrema pobreza, el uso de la fuerza podía aterrorizar a algunos por cierto tiempo, pero reproducía y multiplicaba necesariamente los líderazgos de los violentos, reduciendo a su vez la legitimidad de quienes la aplicaban. El comentario del General Heleno, primer jefe militar de la Minustah sobre el carácter esencialmente pacífico de los haitianos y que "con estos niveles de miseria y diferencia social otros países latinoamericanos se harían socialmente incontrolables», era menos una condonación de la violencia de los bandidos que una protesta implícita por los niveles de miseria circundantes. La opinión resultó irritante para algunos haitianos acomodados que sentían que su situación era similar -como ellos mismos lo indicaban- a la de los tutsis frente a los hutus en la tragedia de Rwanda, pero reflejaba de manera muy adecuada la reacción latinoamericana ante las enormes diferencias sociales y la abismante miseria de las masas haitianas. De tal manera que la idea del desarrollo económico como factor vinculado a la reducción de la violencia, de la generación de empleo, de una política de obras publicas, del respeto a las organizaciones de base en las poblaciones marginales, del diálogo con sus dirigentes, y por lo tanto de un rechazo a la demonización que normalmente se hacía de los ex dirigentes aristidianos, eran todos conceptos que derivaban naturalmente de la visión que los latinoamericanos tenían de la lucha contra la violencia.

A estos conceptos se agregaría más tarde la consideración política de que la represión indiscriminada hacia las bandas criminales, así como de acoso a quienes de una u otra manera se vinculaban a ellas, conduciría necesariamente a la destrucción de una elección plenamente participativa y legítima, cuyos resultados fueran considerados correctos tanto dentro como fuera del país. De aquí se derivaban consecuencias políticas prácticas a las que nos referiremos más adelante.

Estas normas políticas que guiaron a la Misión no constituyeron un asunto menor ni menos aún simplemente formal. Significaron básicamente que la definición de la Misión de Paz privilegió los aspectos políticos más que los militares. Ni los ex militares haitianos ni las bandas armadas de las poblaciones marginales constituían una fuerza capaz de desafiar militarmente al contingente de las Naciones Unidas. En cambio, la confrontación de actividades criminales organizadas constituía un desafío político particularmente grave para una misión de las características de la Minustah, y requería de una respuesta elaborada y de una distinción entre fases claramente establecidas. No de una vasta operación bélica sin espacio político 
definido. Al mismo tiempo, mostraba un cambio de concepto de una Misión originalmente concebida para tratar «un Estado fallido", es decir, un peligro a la seguridad regional, a otra bien distinta, que enfocaba una sociedad latinoamericana en condiciones de extrema pobreza, y por lo tanto se constituía en torno a conceptos de "desarrollo económico", «diálogo nacional», reconstitución de tejido social y relanzamiento de instituciones políticas ${ }^{4}$.

\section{LA «COMPRENSIÓN POLÍTICA» DEL PROCESO HAITIANO}

La Minustah fue la segunda Misión militar instalada en Haití tras el derrocamiento del Presidente Jean Bertrand

4 No podemos examinar este tema con la extensión que se debería. Baste decir que la opción adoptada se distanciaba notoriamente de los conceptos a la sazón predominantes en Washington acerca de «Estados fallidos». Tal como ha señalado Juan Gabriel Tokatlian, en los Estados centrales se desarrolló durante las ultimas décadas una verdadera "aproximación esquizofrénica» ante los Estados periféricos. Se les pedía desmontar el Estado económico, pero fortalecer el de seguridad. Y en este campo, los remedios socioeconómicos o de construcción de legitimidad institucional eran, en el mejor de los casos, solo un acompañamiento. «La atención, en términos conceptuales, burocráticos y recursivos, se colocó en la dimensión militar». Véase «La construcción de un «Estado fallido» en la política mundial: el caso de las relaciones entre Estados Unidos y Colombia», por Juan Gabriel Tokatlian, Buenos Aires, agosto de 2007.
Aristide. La primera, que permaneció tres meses, debía separar a los bandos contendientes e impedir una masacre. A la Minustah le correspondía mantener la paz, estabilizar el país y ayudar al gobierno provisional a reconstituir un sistema político. Para ello debía procurar restablecer el diálogo político entre las partes que se acababan de confrontar, pacificar los espíritus, destacar entre las élites, aquellas personalidades más constructivas y menos comprometidas con ese pasado reciente y violento que muchos se negaban a abandonar. Por sobre todo, debía «comprender» el proceso político.

Pero contra esa buena comprensión se erigían los prejuicios de casi todos los que la recibían, las historias de fracasos individuales y nacionales en experiencias previas, los intereses virulentos contrapuestos, y la falta prácticamente completa de información acerca de cómo pensaban y sentían los haitianos comunes. En efecto, la Misión llegó a Haití acompañada del pesimismo de todos los «expertos en Haití», quienes en la academia o en la política, y especialmente-aunque no solamente-desde Washington, predecían una nueva catástrofe de la comunidad internacional en lo que muchos denominaban «the country of infinite impossibilities». Si esta visión no se impuso fue en buena parte por la ausencia relativa de los Estados Unidos de la conducción de la Misión. La guerra de Irak, y una «fatiga» en Washington con el tema haitiano, en que el peso de una historia de fuertes compromisos seguidos 
invariablemente de fracasos, generaba un infinito pesimismo, llevó a la vieja guardia de veteranos de Haití a esperar que los latinoamericanos tuvieran la capacidad de imaginar soluciones nuevas. Por otra parte, la creación por la misma Resolución 1442 del Consejo de Seguridad de un «core group» de países y organismos internacionales participantes en la Misión, -en el cual los latinoamericanos tuvieron un rol muy importante-, homogeneizaron opiniones entre ellos, los Estados Unidos, los países Europeos y Canadá, generándose así en definitiva un consenso acerca de la prioridad que debía otorgarse al proceso político haitiano.

Llegó también esta Misión a Haití acompañada de historias que no respondían a la situación actual del país. La indicación transmitida desde Washington a las Naciones Unidas acerca del rol principalísimo de los ex militares haitianos en la subversión, y el riesgo militar que estos constituían para la Misión, fue determinante para la distribución de los cascos azules en el territorio, pero fue al mismo tiempo un error producto de una lectura atrasada de los acontecimientos. Efectivamente, los individuos que provenían del Ejército haitiano disuelto por Aristide pretendían reemplazar a la policía y durante algunos meses fueron capaces de montar dos o tres operaciones sorpresivas contra aquella, asaltando sus cuarteles y expulsando a sus moradores. Sin embargo, fueron siempre incapaces de hacer frente a los soldados de las Naciones Unidas. En poco tiempo habían sido desarticulados, o se habían rendido e incorporado a programas de reinserción.

En cambio, la falta de información acerca de las bandas armadas de las poblaciones marginales de las ciudades estableció un problema mayor para la Misión. La naturaleza de estos grupos, su grado de imbricación con la población civil, su nivel real de armamento y la relación entre criminalidad y política y muy particularmente entre tráfico de drogas y política, constituyeron en un inicio problemas intratables, en ausencia como se estaba de un servicio de inteligencia adecuado. La contribución de la policía haitiana fue en este campo prácticamente nula. Durante los primeros meses, las tropas de la Minustah debieron a menudo quitar los teléfonos celulares a los oficiales policiales para impedir que estos advirtieran a los ex militares o a veces a los propios bandidos la inminencia de una operación. La tarea de armar un servicio de inteligencia propio de la Misión, capaz de apoyar las decisiones políticas y las operaciones militares, tomó más de un año.

Por su parte, los miembros más destacados de la dividida sociedad haitiana desarrollaron un esfuerzo repetido varias veces en el pasado: la cooptación de las misiones extranjeras en favor de sus intereses contrapuestos. Los representantes de las Naciones Unidas fueron cordialmente invitados a encuentros en los cuales recibieron importantes aunque parciales visiones del drama haitiano. La Misión intentó escuchar 
a todos y recoger todos los puntos de vista. Sin aceptar algunos intentos de establecer límites sobre quienes eran sus interlocutores legítimos, la dirección política de la Minustah se reunió con los miembros de la coalición que había derrocado a Aristide, al igual que con los parlamentarios y algunos dirigentes de base de Lavallas. Intentó igualmente, aunque con éxito relativo, constituir un «grupo consultivo informal» en el que se quería turnar a intelectuales y figuras influyentes de las élites haitianas. Si bien jamás se debilitó el interés de cada uno de los invitados por dialogar, la idea de una contribución colectiva generó siempre reticencias entre los interlocutores más frecuentes de la Misión. De la misma manera, la propuesta de un diálogo político nacional, abierto a todos los haitianos, halló resistencias iniciales, y si bien se canalizó luego con altos y bajos a través de la Presidencia de la República, no prosperó nunca más allá de una dimensión formal y burocrática. En cambio, las reuniones de partidos políticos desarrolladas con el apoyo del Primer Ministro, bajo el auspicio de la Minustah y de algunas organizaciones no gubernamentales fueron importantes y productivas y crearon el clima político necesario para unas elecciones sin exclusiones y plenamente participativas.

En 2003, sin embargo, el estado de ánimo de enemigos y partidarios de Aristide era el que se establece entre vencedores y vencidos. Si bien un "Conseil des Sages», nombrado a dedo, había elegido como Primer Ministro a Gerard Latortue, que encarnaba al menos un empeño unificador, era evidente a los ojos de cualquier recién llegado que los capitanes políticos del sector vencedor aspiraban a destruir todo vestigio del Partido Lavallas, a depurar la "administración pública», así como a juzgar, tras encarcelar, a quienes eran sospechosos de haber cometido crímenes o exacciones durante el régimen derrocado. Por su parte, los ex partidarios de Aristide que habían permanecido en el país se hallaban a la defensiva y atemorizados ante una posible persecución, que pareció tornarse real tras el encarcelamiento del ex Primer Ministro Yvon Neptune. Se suponía que este era inmune por haber facilitado el proceso de transición legal entre el Presidente expulsado y Boniface Alexandre, Presidente de la Corte Suprema. Algunos miembros de Lavallas, sin embargo, eran sospechosos de jugar el doble juego de reclamar por sus derechos al mismo tiempo que mantenían contactos y alentaban a líderes de las poblaciones marginales que mantenían relaciones con jefes de gangas, o lo eran ellos mismos.

En este cuadro, la Misión -en complicidad con el gobierno provisional- debía progresivamente alterar el supuesto político de vencedores y vencidos y combatir las políticas de exclusión y revancha. De ahí la necesidad de promover un diálogo nacional. Pero de ahí también los problemas políticos de la Misión. En realidad, persiste curiosamente no solo en Haití sino en muchísimas partes, incluyendo algunas 
Misiones de las Naciones Unidas, la idea de que ingresar a un país con tropas internacionales, y actuar dentro del marco del Capítulo VII de la Carta de las Naciones Unidas, no afecta la soberanía de ese país ni resta independencia a sus actores, pero que en cambio, promover que los partidos políticos o las organizaciones sociales se reúnan, los líderes depongan en beneficio del bien común ambiciones personales, las élites comprendan el drama en el que han puesto a su sociedad, eso sí constituye una intervención inconveniente. En esta curiosa perspectiva, usar la fuerza es legítimo, pero introducirse en el debate interno e intentar orientar y persuadir a favor de opciones políticas de paz es «intervencionismo político». Sin dudas que este prurito hizo más difícil y sobre todo más lento avanzar en los caminos del diálogo y la negociación.

Al mismo tiempo, fue decisivo para la Misión y para Haití que el Primer Ministro Gerard Latortue no abonara jamás esta perspectiva. Más aún, las Naciones Unidas no habrían podido realizar aquellas tareas que contribuyeron al diálogo político, la organización de elecciones y la creación de las condiciones políticas para enfrentar las bandas armadas, de no haber sido por la forma crecientemente inclusiva y antisectaria en que Latortue condujo el gobierno de transición. Sus críticos, que abundan, olvidan la hazaña política que implica ser relevante cuando se está a la cabeza de un gobierno con poder virtual, de un Estado colapsado en un país ocupado por tropas de las
Naciones Unidas y en una sociedad tan violenta como dividida políticamente entre élites acostumbradas a danzar al borde del precipicio. Y el Primer Ministro supo ser relevante -si no decisivo- en los acontecimientos que llevaron a las elecciones presidenciales y parlamentarias y al clima político que se estableció en el país.

\section{El DILEMA HOBBESIANO}

Los espacios abiertos al diálogo político entre los partidos, así como el relativo encauzamiento del consejo electoral -conseguido a pesar de su inoperancia y del carácter pendenciero de algunos de sus miembros- hicieron ver en 2005, tanto al gobierno como a la Misión, que las elecciones presidenciales y parlamentarias se llevarían a cabo a pesar de eventuales retrasos. Bajo la conducción de la OEA y la Minustah, el proceso de inscripción electoral seguía al registro y posterior entrega de una identificación nacional a todos los haitianos, hecho sin precedentes en la historia del país. El pueblo haitiano había concurrido al proceso de registro con admirable paciencia, y a pesar de que las élites de los partidos y los eventuales candidatos presidenciales recurrían permanentemente al uso del potencial «si» al referirse a la realización del proceso electoral, todos se aprestaban a la competencia. Por otra parte, las operaciones de fuerza desarrolladas por la Minustah en julio de 2005 en Cité Soleil, exigidas por la 
brutalidad de los ataques contra los trabajadores del puerto y de la aduana, así como por los frecuentes e indiscriminados atentados a bala contra quienes circulaban en torno al perímetro de la Cité, habían acabado con la principal banda armada de todas las que aterrorizaban, a la vez que reclutaban miembros entre la población más pobre de Puerto Príncipe. Desde septiembre en adelante, la inscripción electoral se había realizado en Cité Soleil sin mayores problemas. $\mathrm{Y}$ a pesar de que la fecha de las elecciones continuaba en entredicho, la población de esa zona parecía responder con la misma tranquilidad e interés que el resto del país al llamado a registrarse para votar. Los jefes de las bandas armadas habían adoptado su máscara de «dirigentes de base» y aceptaban sin problemas que la Minustah y la OEA condujeran el registro de la población. A pesar de las predicciones en contrario, los agentes inscriptores realizaron apaciblemente su trabajo sin riesgos para su seguridad. En esos días de septiembre nada hacía prever lo que vendría.

En efecto, sin causa aparente y de manera sorpresiva, en cuestión de semanas la violencia se apoderó nuevamente de Puerto Príncipe y un inusitado y aparentemente inexplicable número de secuestros y asesinatos llenó a muchas familias de miedo y dolor. Solo en noviembre de 2005 se produjeron más de 200 secuestros y más de una decena de cadáveres aparecieron abandonados en las calles. El gobierno recibió información que apuntaba a la participación de policías y a las redes de narcotraficantes en la ola de crímenes. A su vez, el servicio de inteligencia de la Misión adjudicó su origen a la reacción de criminales y sus aliados policiales ante la lucha contra la corrupción que había desatado Mario Andresol, el nuevo e insobornable jefe policial. Los crímenes parecieron multiplicarse. En realidad, en las condiciones sociales de la ciudad el origen del caos quedaba rápidamente superado por sus propias condiciones de reproducción. $\mathrm{Al}$ acercarse Navidad, la interconexión entre las distintas fuentes y redes criminales pareció hacerse total. Y si bien resultaba siempre riesgoso dar una lectura coordinada a estos hechos, el objetivo de aterrorizar a la población y mostrar la ineficacia de la Minustah era evidente. Los asesinatos de varios soldados del batallón jordano por francotiradores, y la trágica decisión de quitarse la vida del General Bacellar, jefe militar de la Minustah, compusieron un clima en que las presiones para usar la fuerza se hicieron extremadamente difíciles de resistir. Sin embargo, ¿cómo podía conciliarse el objetivo de realizar elecciones con el establecimiento de un estado de sitio y una operación de tipo rastrillo para neutralizar a los criminales?

Pronto surgieron las voces en favor de una indefinida postergación de las elecciones. La predicción de que «en este clima» las elecciones eran imposibles, o constituían una invitación a que los criminales desataran la violencia contra los electores -como había ocurrido en el pasado- se hicieron fre- 
cuentes en el discurso político y en los análisis periodísticos. En un proceso de espiral que los haitianos conocen bien, el caos era alimentado por el pánico y este a su vez por aquel. Los ataques a la Minustah, acusada de inoperancia, se multiplicaron y se transformaron abiertamente en una campaña sistemática de desprestigio. La Misión enfrentó entonces su momento más decisivo: ¿era necesario aplicar la fuerza y "pacificar» el camino de la construcción electoral e institucional, o por el contrario, había que impulsar la construcción de un gobierno legítimo que asegurara las condiciones políticas necesarias para aplicar la fuerza contra los violentos? ¿Qué era primero, la aplicación de la fuerza para permitir la elección, o la elección de un gobierno que permitiera la fuerza? La Minustah se inclinó clara y firmemente por esta segunda opción. Y no se equivocó con ella.

Naturalmente, esta opción traía consecuencias que resultaban altamente polémicas y muy complejas de administrar. En estricto cumplimiento de la Resolución del Consejo de Seguridad, la Minustah afirmó invariablemente y desde el inicio que la reconstrucción del marco institucional, y el proceso electoral, requerían la participación de quienes habian sido derrotados junto a Aristide. Sin ellos, el proceso carecería completamente de legitimidad. Era para todos evidente que esta perspectiva a lo menos contrariaba a aquella parte de la elite haitiana que tan pocos meses antes había visto al alcance de la mano una victoria total sobre el odiado y temido régimen del ex sacerdote salesiano. La idea de un «cambio de curso histórico» con la que varios dirigentes haitianos opositores habían acogido la transición tenía como elemento central «el fin de la impunidad», lo que en la práctica significaba la proscripción del partido Lavallas, o el encarcelamiento de sus principales dirigentes. El paulatino desinterés del gobierno de Latortue en proseguir un camino de confrontación reflejó no solo un sentido de lo posible y lo prudente, sino también las convicciones que animaban el trabajo de la Minustah y la voluntad de la comunidad internacional.

El dramatismo de la situación en noviembre y diciembre de 2005 puso sin embargo las cosas en una dimensión aún más compleja. Ya no se trataba solo de garantizar el derecho de los dirigentes de Lavallas o de cualquier otro partido o movimiento a participar en el proceso electoral, sino también de asegurar que la población de aquellos barrios que parecían identificarse con el derrocado presidente participara en un ambiente de mínimas condiciones de seguridad. ¿Cuál sería la percepción de una elección en la que los habitantes de Cité Soleil -transformada para bien o mal en un símbolo del drama haitiano- no podían votar? ¿Cómo podría invitarse a participar electoralmente durante el día a personas que vivían cada noche enfrentamientos a bala entre soldados de la Minustah y criminales armados? La lógica más elemental indicaba que la Misión debía suspender todas las operaciones militares en los barrios 
más pobres de la ciudad, por más que esto significara entregar a las bandas armadas un certificado de tregua.

El resultado del 7 de febrero es conocido. Más allá de todas las dificultades, el pueblo haitiano concurrió a votar masivamente en un día que transcurrió sin episodios de violencia. La victoria de René Preval fue categórica. La demora en la entrega de los resultados y la polémica en torno a la forma de conteo de los votos blancos no desmerecieron un proceso electoral de una corrección y limpieza superior a cualquier otro en la historia del país.

Pocos meses más tarde el Presidente Preval ofreció a los líderes de las bandas armadas una negociación, recibiéndoles en el Palacio presidencial e indicándoles sin ambages que debían entregar las armas y proceder al proceso de desarme de sus hombres que había establecido la Minustah. La alternativa, según señaló el Presidente, era «desarmarse o mo-
rir».La población de Cité Soleil pudo percibir cómo la autoridad estatal ofrecía a los líderes de las organizaciones criminales un camino de solución, que implicaba su rendición, pero también el reconocimiento de sus derechos. La negativa de los líderes de las bandas a aceptar esta oferta y la reanudación de la violencia en la ciudad condujo al Presidente a solicitar formalmente a la Misión la neutralización de todas las bandas armadas. En un clima político marcado por el consenso político y una nueva política estatal hacia las poblaciones más afectadas por las actividades criminales, los soldados de la Misión se encontraron con organizaciones criminales que habían perdido el apoyo de la población. La desaparición de las bandas armadas y la apertura de Cité Soleil marcaron una nueva fase en la historia del proceso político y de desarrollo haitiano. 